\title{
Fractal Simulation on Random Rough Surface
}

\author{
Jian Wang, Chengbao Wu, Chuansheng Liu, Jing Wei \\ School of Aircraft Maintenance and Engineering, Guangzhou Civil Aviation College, Guangzhou \\ 510430, China
}

Keywords: Rough surface, Random; fractal, simulation

\begin{abstract}
Two kinds of multi-dimensional fractal functions were introduced, and the VB program was wrote based on the rule of the fractal functions, the programming operation to the simulation on the random rough surface was realized to display the randomness of rough surface visually. The results indicated that the simulation surface could reflect the random characterization of surface roughly.
\end{abstract}

\section{Introduction}

Surface simulation has been one of important research interests in many fields of science. For example, the materials science and engineering, the tribology, the computer graphics and the geophysics, etc.. The models of the fractal simulation can be used to measure the surface parameters of the true surface replacing the manual surface, but also simulate the random phenomena of the surface, which can't be controlled by the experiments. The fractal simulation on the surface was limited in the simulation of profile curve of the true surface in the early stage. The main fractal models proposed in this stage included the W-M fractal function model, structure function model and fractal interpolation function model. The three models mentioned above can be used to evaluate the fractal characteristic of the surface based on the limited points on the surface profile curve, and are efficiency in describing the continuity of the surface. So, all the models are ideal tools for investigating the self-similarity of the surface profile curve. But, the models can only characterize the two-dimensional morphology of the surface, and investigate the two-dimensional characteristics of the surface. With the development of the fractal theory and the study on the fractal curved surface, high-dimension fractal functions were proposed to simulate the three-dimensional structure of the surface.

When using the structure function to calculate the fractal dimension of the profile curve of the surface, one can find that the value of the fractal dimension is in a range from 1 to 2 [1]. The value of the fractal dimension of the surface calculated by the projective covering method (PCM) ranges from 2 to 3[2, 3, 4]. In this work, the authors simulated the morphology of surface by using the two different high-dimension fractal function, Ausloos-Berman fractal function(A-B fractal function) and Manjumdar-Tien fractal function(M-T fractal function).

\section{Fractal functions}

The Weierstrass-Mandelbrot fractal function (W-M fractal function) was proposed by Weierstrass in 1872, and then modified by Mandelbrot who devised the fractal theory in 1977. This function can be used to get the spectrogram of the profile curve and produce manual profile curve with uncertainty. At the same time, it could also be used to simulate the profile curve of the true surface. So it is used widely. Its complex function is [1]:

$$
w(x)=\sum_{-\infty}^{\infty} r^{(D-2) n}\left(1-e^{i r^{n} x}\right) e^{i \phi_{n}}
$$

Where $w$ is the complex function of the variable $x ; r$ is a parameter controlling the spatial frequency density of the profile curve of the surface, $r>1$, it is always 1.5 for the factors of surface smoothness and frequency distributional density ${ }^{[4]}$; $D$ is taken to be the fractal dimension of the profile curve of the surface, $1<D<2$, as the $D$ is much closer to 1 , it means that profile curve of the surface is much smoother, as $D$ is much closer to 2, it means that the profile curve of the 
surface is much coarser; $n$ is frequency index; $\varphi_{n}$ is arbitrary phase, which can be chosen to make $w$ exhibit deterministic or stochastic behavior.

The real part of the fractal function can be used to describe the fractal profile curve of the surface. As shown in the Eq. 2:

$$
\begin{aligned}
Z(x) & =\operatorname{Re}(w(x)) \\
& =\sum_{n=-\infty}^{\infty} r^{(D-2) n}\left[\cos \varphi_{n}-\cos \left(r^{n} x+\varphi_{n}\right)\right]
\end{aligned}
$$

Where all the parameters in Eq. 2 are same with those in Eq.1.

With the development of fractal theory, we find that the theory can be used to study the fractal curve, but also to evaluate the fractal surface, and many fractal functions were devised. Among these functions, the Ausloos-Berman fractal function (A-B fractal function) and Manjumdar-Tien fractal function (M-T fractal function) are classical.

\subsection{Ausloos-Berman fractal function (A-B fractal function).}

In 1985, Ausloos and Berman developed a W-M fractal function with many variables based on the W-M fractal function with one variable to describe the high-dimensional random process [5]:

$$
w(r)=\left(\frac{\ln r}{M}\right)^{1 / 2} \sum_{m=1}^{M} A_{m} \sum_{n=-\infty}^{\infty}\left[1-\exp \left(i k_{0} r^{n} \hat{n}_{m} \cdot r\right)\right] \exp \left(i \phi_{m, n}\right)\left(k_{0} r^{n}\right)^{D_{s}-(d+1)}
$$

The real part of the fractal function can also be used to describe the fractal profile curve of the surface. Its polar coordinate is [5]:

$Z(\rho, \theta)=\operatorname{Re}(W(r))$

$$
=\left(\frac{\ln r}{M}\right)^{1 / 2} \sum_{m=1}^{1} A_{m} \sum_{n=-\infty}^{\infty}\left(k r^{n}\right)^{\left(D_{s}-3\right)}\left\{\cos \varphi_{m, n}-\cos \left[k r^{n} \rho \cos \left(\theta-\alpha_{m}\right)+\varphi_{m, n}\right]\right\}
$$

Where $\rho$ and $\theta$ are the plane polar coordinates of the point at the height $Z$ of the surface, which can be expressed by the $x$ and $y$ in rectangular coordinate system:

$$
\begin{aligned}
& \rho=\left(x^{2}+y^{2}\right)^{1 / 2} \\
& \theta=\tan ^{-1}(y / x)
\end{aligned}
$$

Where the physical meaning and the order of magnitude of the $r$ is same with the one in Equ.(1). $D_{S}$ is the surface fractal dimension, $2<D_{s}<3$, it can be obtained by the methods of the power spectral method and projective covering method. The $D_{S}$ is much larger, the occupation space of the coarse surface is much larger, and the fine structure and spatial levels in the surface are much more. $M$ is the number of space fluctuation in simulating the fractal surface. As for the isotropy surface, $A_{m}$ does not change with the changing $m$ :

$$
A_{m}=2 \pi(2 \pi / G)^{\left(2-D_{s}\right)}
$$

Where $\mathrm{k}$ is the wave number relevant to the sampling length $\mathrm{L}, k=2 \pi / L ; \mathrm{n}$ is frequency index, its minimum value is $1 / L$. So, the lower limit value of the $k$ can be set as 0 , and the shortest wave length is not much less than $L_{s}$, the up limit value of the $k$ can be expressed as:

$$
n_{\max }=\operatorname{int}\left[\frac{\log \left(L / L_{s}\right)}{\log r}\right]
$$

Where int $[\cdots]$ is the maximum value in the square brackets. $a_{m}$ is the peak value for compensating for the azimuth angle, and $\alpha_{m}=\pi m / M . \phi_{m, n}$ obeys the uniform distribution in the range $[0,2 \pi]$.

To substitute the $\rho, \theta, A_{m}, \alpha_{m}$ and $k$ into Eq.4, one can obtain: 


$$
\begin{aligned}
& Z(x, y)=L\left(\frac{G}{L}\right)^{\left(D_{s}-2\right)}\left(\frac{\ln r}{M}\right)^{1 / 2} \\
& \sum_{m=1}^{M} \sum_{n=0}^{n_{m x}} r^{\left(D_{s}-3\right) n} \times\left\{\cos \varphi_{m, n}-\cos \left[\frac{2 \pi r^{n}\left(x^{2}+y^{2}\right)}{L} \times \cos \left(\tan ^{-1}\left(\frac{y}{x}\right)-\frac{\pi m}{M}\right)+\varphi_{m, n}\right]\right\}
\end{aligned}
$$

Where $Z(x, y)$ is the height function of the surface, it was used to simulate different anisotropic randomness surface. $G$ is the height scaling parameter irrelevant to the frequency. In this function, $G$ and $D$ are variables, which can be obtained by experiments.

After Mandelbrot, Yan [6] simulated the contact fractal surfaces with the fractal dimensions 2.4 and 2.8 respectively. Wu [7] simulated the theoretical fractal surface. Zhang Yi [8] simulated the surface of the optical switch of the Micro Electro Mechanical System (MEMS), the results indicated that the morphology of the simulated surface is similar with the image of the true surface token by scanning tunneling microscope (STM).

\subsection{Manjumdar-Tien fractal function (M-T fractal dimension)}

Manjumdar and Tien [9] set up the simulation function by building the relationship between the profile spectrum and surface spectrum:

$$
Z(x, y)=F(D) G^{(D-1)} \sum_{n=n_{L}}^{\infty} \frac{\cos 2 \pi \gamma^{n}[x+g(y)] \cos 2 \pi \gamma^{n}[y+g(x)]}{\gamma^{(2-D) n}}
$$

Where $1<D<2, \gamma>1$, and $F(D)$ is a function relevant to $D$, which can be expressed as:

$$
F(D)=\left(\frac{2 \ln (5-2 D)(7-2 D) E(D)}{\pi}\right)^{1 / 2}
$$

$D$ is the fractal dimension of the profile curve, $D_{s}=D+1$;

$$
E(D)=\int_{0}^{2 \pi}\left\{\cos ^{(5-2 D)} \theta-\cos ^{(7-2 D)} \theta\right\} d \theta
$$

As for fractional Brownian surface, $E(1.5)=\pi / 16$.

$g(a)$ is a function making the phase of the surface random:

$$
g(a)=C \sum_{n=n_{L}}^{\infty} \frac{\cos 2 \pi r^{n} a}{r^{(2-D) n}}
$$

The Eq.11 generates a Gaussian distribution signal with standard deviation. The $C$ in Eq.13 can be expressed as:

$$
C=\frac{1}{2 \times 2^{1 / 2}} \frac{r^{(2-D)\left(n_{L}-1\right)}}{\left[r^{(4-2 D)}-1\right]^{1 / 2}}
$$

Komvopoulos and Ye [10] simulated the interface between the slider and the disc with the fractal dimension 2.44. Zhou [11] characterized the wear surface and wear degree by using of the function. Chen Hui [12] simulated the surface by this function, the results indicated that the simulation surface obeyed the Gauss distribution and it was suitable for surface simulation.

\subsection{Comparative analysis of the two fractal functions}

It can be found from the physical meaning of the fractal functions mentioned above that both Eq.9 and Eq.10 are suitable for simulating the fractal surface. The difference between the two functions is that the Eq.9 can be used to simulate both the three-dimension random isotropic surface and the three-dimension random anisotropic surface, but one must calculate the fractal dimension (Ds) of the surface firstly; for the fractal function, as shown in Eq.10 and Eq.14, when it is used to simulate the fractal surface, one should calculate fractal dimension of the profile curve of the surface (D), which is in the range from 1 to 2, and then substitute the D+1 into the Eq.10 and Eq.14 to simulate the surface. 
The simulation process was realized by the VB computer language. The steps for writing the VB procedure are as following:

Step1: Design the interface form, and load the needed control.

Step2: Set the button of the number of input row to control the number of the data.

Step3: Set the button of the number of the parameters to set the number of the parameters.

Step4: Set the button of the calculate to run the computing procedure.

Step5: Set the button of the down load to export the data to the Excel.

Step6: Test the procedure and modify it.

\section{Fractal simulation on the surface}

The surfaces with fractal dimensions 2.0894 and 2.6375 were simulated by the A-B fractal function and M-T fractal function respectively based on the VB language procedure. The simulation surfaces are shown in Fig.1. The Fig.1 (a) shows the simulation surface of the surface with fractal dimension 2.0894 based on A-B fractal function. The simulation surface of the surface with fractal dimension 2.6375 by the M-T fractal dimension is shown in Fig. 1 (b).

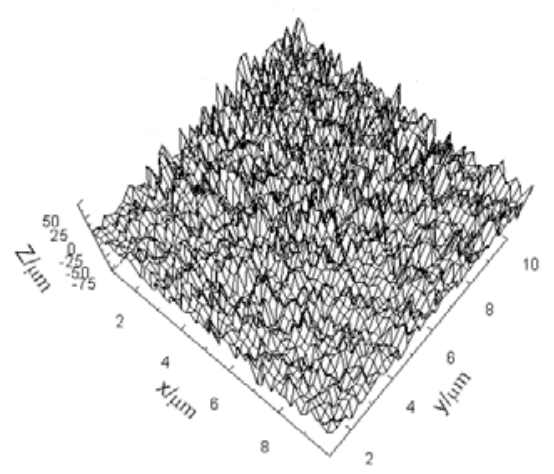

(a)

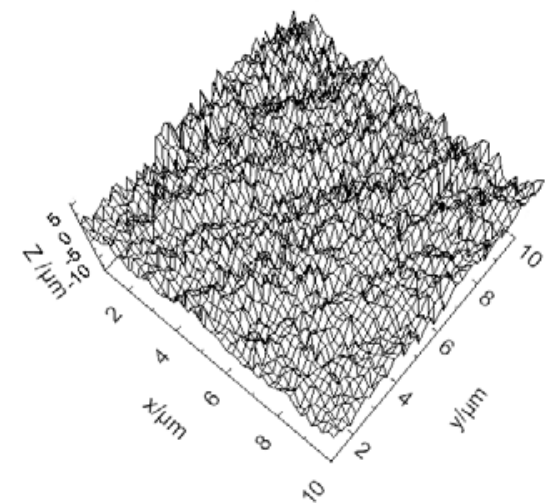

(b)

Fig.1 Simulation surface with different dimensions:(a) on A-B fractal function $\left(D_{S}=2.0894\right)$,

(b)on M-T fractal function $\left(D_{S}=2.6375\right)$

Fig.2 (a) and (b) show the gray images of the simulation surface (as the Fig.1 shows) and the profile curves at the any positions. The number 1 in the Fig. 2 means gray image of the simulation surface, the number 2 means the profile curve of the gray image at the any horizontal position, and the number 3 means the profile curve of the gray scale image at any vertical position.

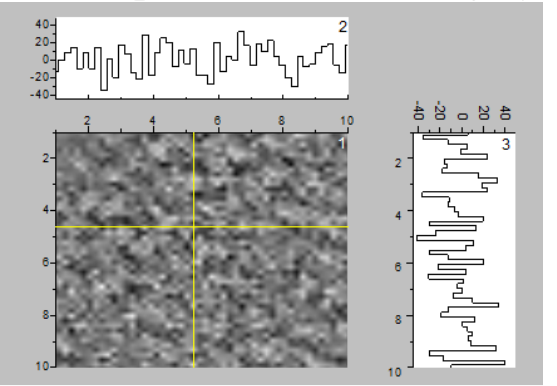

(a)

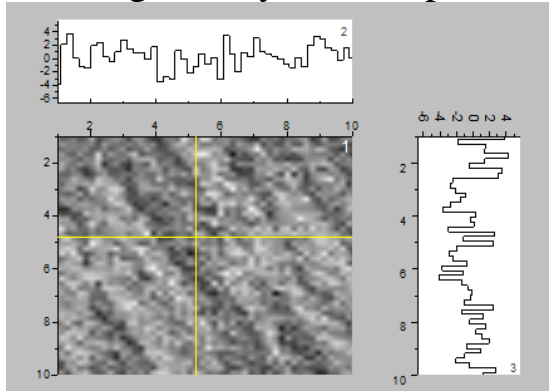

(b)

Fig.2 gray image of simulation surface with different dimension:(a) based on A-B fractal function $\left(D_{S}=2.0894\right)$, (b) on M-T fractal function $\left(D_{S}=2.6375\right)$

It can be seen easily from Fig. 1 that both of the two fractal functions can be used to simulate random rough surface with different degree of roughness. But there are some differences between the simulation surfaces. Firstly, concave-convex feeling, and ups and downs of feeling of the simulation surface in Fig.1 (b) is more evident than those in Fig.1 (a); secondly, the height of the point on the simulation surface in Fig.1(a) is in a range from -5 to $5 \mu \mathrm{m}$, the amplitude value is only $10 \mu \mathrm{m}$, while the height of the point on the simulation surface in Fig.1(b) is range from -45 to $49 \mu \mathrm{m}$, the amplitude value is close to $95 \mu \mathrm{m}$. From Fig.2, one can find that the profile curves of simulation surface at any horizontal and vertical position based on gray scale image are random 
distribution, everywhere continuous and not differentiable, and have fractal characteristics. Zhu [13] investigated the simulation on the profile curve of the surface; the results indicated that the less the fractal dimension is, the simpler the simulation profile curve is, and the larger the amplitude value is, that is, the surface is much rougher. On the contrary, the larger the fractal dimension is, the more complex the simulation profile curve is, the less the amplitude value is, that is, the surface will be much smoother. Therefore, the simulation surface by fractal function in this work can characterize the true surface approximately.

\section{Conclusions}

The simulation surface can reflect the degree of the roughness of the true surface. Especially, the gray scale image of simulation surface is close to that of the true surface. But, the simulation surface is generated by the fractal function, while the true surface is not rigidly fractal.

\section{References}

[1] Berry M.V., Lewis Z.V., On the Weierstrass-Mandelbrot fractal function. Proceedings of the Royal Society of London.Series A, Mathematical and Physical Sciences. London, the Great Britain: the Royal Society, 370(1743): pp459-484, 1980.

[2] Wu Chengbao, Liang Jizhao, Fracture-surface fractal dimension of PP/diatomite composites and its relation to impact strength. Journal of South China University of Technology (Natural Science Edition), 37(8): pp150-154, 2009.

[3] Liang Jizhao, Wu Chengbao, Study on relationship between impact strength and fracture surface fractal dimension of PP/Nano-CaCO 3 composites. Materials Engineering, 54(10), pp53-56, 2009.

[4] Yan W., Komvopoulos K.A., fractal analysis of stiction in microelectromechanical systems. Journal of Tribology, 119(3), pp391-400, 1997.

[5] Ausloos M., Berman D.H., A multivariate Weierstrass-Mandelbrot function. Proceedings of the Royal Society of London.Series A, Mathematical and Physical Sciences. London, the Great Britain: the Royal Society, 400(1819), pp331-350, 1985.

[6] Yan W, Komvopoulos K., Contact analysis of elastic-plastic fractal surfaces. Journal of Applied Physics, 84(7),pp3617-3624, 1998.

[7] Wu J. J., Characterization of fractal surfaces. Wear, 239(1), 36-47, 2000.

[8] Zhang Yi, Luo Yuan, Xu Xiaodong, Analysis and simulation for micro-mirror of MEMS optical switch based on fractal theory. Semiconductor Optoelectronics, 27(6): 672-674, 2006.

[9] Majumdar A, Tien C.L., Fractal characterization and simulation of rough surfaces . Wear, 136(2),pp 313-327, 1990.

[10] Komvopoulos K., Ye N., Three-dimensional contact analysis of elastic-plastic layered media with fractal surface topographies. AMSE Journal of Tribology, 123, pp632-640, 2001.

[11] Zhou G., Leu M.C., Blackmore D., Fractal geometry modeling with applications in surface characterization and wear prediction. Journal of Machine Tools and Manufacture, 35, pp203-209, 1995.

[12] Chen Hui, Hu Yuanzhong, Wang Hui, Simulation and characterization of fractal rough surfaces. Chinese Journal of Mechanical Engineering, 42(9), pp219-223,2006.

[13] Zhu Hua, Ge Shirong, Comparison of fractal characterization effects of structure function and mean square root. Journal of China University of Mining and Technology, 33(4), pp396-399,2004. 